\title{
Exonic splicing enhancers in fission yeast: functional conservation demonstrates an early evolutionary origin
}

\author{
Christopher J. Webb, Charles M. Romfo, Willem J. van Heeckeren, and Jo Ann Wise ${ }^{1}$ \\ School of Medicine, Department of Molecular Biology and Microbiology, Case Western Reserve University, \\ Cleveland, Ohio 44106-4960, USA
}

\begin{abstract}
Discrete sequence elements known as exonic splicing enhancers (ESEs) have been shown to influence both the efficiency of splicing and the profile of mature mRNAs in multicellular eukaryotes. While the existence of ESEs has not been demonstrated previously in unicellular eukaryotes, the factors known to recognize these elements and mediate their communication with the core splicing machinery are conserved and essential in the fission yeast Schizosaccharomyces pombe. Here, we provide evidence that ESE function is conserved through evolution by demonstrating that three exonic splicing enhancers derived from vertebrates (chicken ASLV, mouse IgM, and human cTNT) promote splicing of two distinct $S$. pombe pre-messenger RNAs (pre-mRNAs). Second, as in extracts from mammalian cells, ESE function in $S$. pombe is compromised by mutations and increased distance from the 3 '-splice site. Third, three-hybrid analyses indicate that the essential SR (serine/arginine-rich) protein Srp2p, but not the dispensable Srp1p, binds specifically to both native and heterologous purine-rich elements; thus, Srp2p is the likely mediator of ESE function in fission yeast. Finally, we have identified five natural purine-rich elements from $S$. pombe that promote splicing of our reporter pre-mRNAs. Taken together, these results provide strong evidence that the genesis of ESE-mediated splicing occurred early in eukaryotic evolution.
\end{abstract}

[Keywords: ESE; U2AF; SR protein; Srp1p; Srp2p; ASF/SF2]

Supplemental material is available at http://www.genesdev.org.

Received September 27, 2004; revised version accepted November 11, 2004.

Pre-messenger RNA (pre-mRNA) splicing in all eukaryotes requires conserved intronic sequence elements located at the 5'- and 3'-splice sites and branchpoint for both accurate recognition of exon/intron boundaries and catalysis of the two transesterification reactions. Nevertheless, it was appreciated early on that intronic signals alone do not suffice for efficient splicing of some vertebrate pre-mRNAs (Reed and Maniatis 1986; Nelson and Green 1988). The discovery that positively acting elements within exons designated exonic splicing enhancers (ESEs) promote splicing of upstream introns exposed additional layers of complexity in metazoan 3'-splice site selection (Black 2003). ESEs are found both in premRNAs that are constitutively spliced (Mayeda et al. 1999; Schaal and Maniatis 1999) and those that are subject to regulated splicing (e.g., Ryner and Baker 1991; Tian and Maniatis 1992). A large and growing body of evidence indicates that exonic splicing enhancers are widely distributed among metazoans from flies to hu-

${ }^{1}$ Corresponding author.

E-MAIL jaw17@case.edu; FAX (216) 368-2010.

Article published online ahead of print. Article and publication date are at http://www.genesdev.org/cgi/doi/10.1101/gad.1265905. mans (Black 2003). Their importance is underscored by the finding that mutations within ESEs are responsible for the aberrant splicing profiles of pre-mRNAs implicated in disease including SMN2 (Lorson and Androphy 2000; Cartegni and Krainer 2002), HIV-1 env (Caputi and Zahler 2002), FBN1 (Caputi et al. 2002), CD44 (GalianaArnoux et al. 2003), and CFTR (Cartegni et al. 2002; Aznarez et al. 2003).

Among the exonic splicing enhancers identified and characterized experimentally in metazoans, purine-rich ("GAR-type") elements constitute the most abundant class (Cartegni et al. 2002). However, functional selection strategies have revealed not only degenerate purinerich elements (Liu et al. 1998; Schaal and Maniatis 1999), but other classes of ESEs with distinct consensus sequences as well (Coulter et al. 1997; Schaal and Maniatis 1999). More recently, the application of computational strategies has led to the conclusion that sequences capable of enhancing splicing are remarkably common in the exons of higher eukaryotic genes (Fedorov et al. 2001; Fairbrother et al. 2002; Zhang and Chasin 2004). The degeneracy of these motifs most likely represents an evolutionary solution to the problem of how to modulate the composition of exons to ensure accurate and 
efficient splicing while maintaining protein-coding capacity.

Several classes of ESEs have been shown to bind SR (serine/arginine-rich) proteins (Lavigueur et al. 1993; Sun et al. 1993; Tian and Maniatis 1993). The mammalian SR protein family consists of 10 core members, not including isoforms (Screaton et al. 1995) or related genes (Tupler et al. 2001). The core SR protein domain structure consists of an N-terminal RRM (RNA recognition motif) and a C-terminal RS domain; some members also contain a RRMH (RNA recognition motif homology) or zinc finger located between the two identifying domains (Graveley 2000). SR proteins are modular, with RNA binding attributed to the RRM and the RS domain functioning as a splicing activator (Graveley and Maniatis 1998). Roles for SR proteins in both constitutive and regulated splicing have been described and studied extensively in metazoans (Graveley 2000; Hastings and Krainer 2001; Black 2003). Based on their ability to simultaneously interact with the U1-70K protein of the U1 snRNP (small nuclear ribonucleoprotein particle) and the small subunit of the U2AF (U2 snRNP auxiliary factor) heterodimer, SR proteins are proposed to play a central role in a protein-protein interaction mechanism for splice-site pairing across both introns and exons (Hoffman and Grabowski 1992; Wu and Maniatis 1993).

Multiple mechanisms have been proposed to account for how binding of SR proteins to ESEs leads to activation of splicing. The first model, which is based on studies with Drosophila Doublesex (dsx) pre-mRNA, postulates that a complex containing SR proteins, Tra, and the SR-related protein Tra2 assembles on an element in the downstream exon and recruits U2AF to the suboptimal 3'-splice site (Lynch and Maniatis 1996; Zuo and Maniatis 1996). The linchpin of this model is an interaction between SR proteins and the U2AF small subunit $\left(\mathrm{U} 2 \mathrm{AF}^{\mathrm{SM}}\right)$, which are proposed to form a bridge between ESEs and the U2AF large subunit (U2AF ${ }^{\mathrm{LG}}$ ) (Zuo and Maniatis 1996). In support of this model, several studies demonstrated increased $\mathrm{U}_{2} \mathrm{AF}^{\mathrm{LG}}$ binding to 3 '-splice sites in an ESE-dependent manner in nuclear extracts (Wang et al. 1995; Zuo and Maniatis 1996; Graveley et al. 2001). However, other studies failed to find increased ESE-dependent U2AF cross-linking (Guth et al. 1999; Kan and Green 1999; Li and Blencowe 1999), possibly due to different experimental conditions (Guth et al. 2001). In one instance, an ESE promoted splicing by counteracting the negative effect of a downstream splicing silencer (Kan and Green 1999; Shen et al. 2004a). Another alternative to the SR/U2AF protein-protein interaction model for ESE-mediated splicing activation is based on a recent report that a tethered RS domain specifically contacts the branchpoint (Shen et al. 2004b). As proposed for the RS domain of the U2AF large subunit (Valcarcel et al. 1996), this model postulates that SR proteins facilitate annealing of U2 snRNA (Graveley 2004; Shen et al. 2004b). This model is unlikely to be universal, however, as the RS domain is dispensable in some cases of ESEdependent splicing, at least in vitro (Zhu and Krainer 2000; Tange and Kjems 2001).
In the fission yeast Schizosaccharomyces pombe, 4730 introns are distributed among $43 \%$ of the 4972 annotated open reading frames (Wood et al. 2002). S. pombe premRNAs exhibit complex exon/intron architecture, with $>1000$ genes harboring multiple introns (Wood et al. 2002). In contrast, $4.3 \%$ of the 5804 genes in the budding yeast Saccharomyces cerevisiae encode spliced pre-mRNAs; with two exceptions, a single intron is present, generally located near the $5^{\prime}$-end (Spingola et al. 1999). Based on their high degree of degeneracy, the cis-acting signals that direct splicing in $S$. pombe, particularly the branchpoints (Kuhn and Kaufer 2003), are considered to be more similar to their counterparts in multicellular eukaryotes than in S. cerevisiae (Wentz-Hunter and Potashkin 1995). Functional parallels have also been demonstrated; for example, polypyrimidine tracts located between the branchpoint and 3 '-splice site in mammals and fission yeast function prior to the first step of splicing (Reed 1989; Romfo and Wise 1997), whereas a similarly located U-rich signal in S. cerevisiae is required only for the second step (Patterson and Guthrie 1991).

Importantly, S. pombe contains clear orthologs of the trans-acting factors known to recognize and respond to ESEs, namely both subunits of U2AF and an essential SR protein (Srp2p); these factor are not only conserved, but indispensable for life (Potashkin et al. 1993; WentzHunter and Potashkin 1996; Lutzelberger et al. 1999; Romfo et al. 1999; Webb and Wise 2004). Moreover, we have recently shown that $S$. pombe U2AF ${ }^{S M}$ (Uaf2p) and Srp2p interact specifically (Webb and Wise 2004), as do their mammalian counterparts (Wu and Maniatis 1993; Zuo and Maniatis 1996). Budding yeast, on the other hand, contains a degenerate U2AF large subunit, no protein related to the U2AF small subunit (Kaufer and Potashkin 2000), and an SR-like protein that shares features with this family but lacks a specific metazoan ortholog (Siebel and Guthrie 1996).

In this report, we demonstrate that ESE-mediated splicing is not exclusive to multicellular eukaryotes. To investigate enhancer function in $S$. pombe, we first developed reporter constructs to test heterologous ESEs for the ability to promote splicing in vivo. Remarkably, elements from vertebrates, which diverged from fission yeast 1 billion to 1.2 billion years ago (Sipiczki 2000; Wood et al. 2002), function in this unicellular eukaryote with sequence and distance constraints similar to those observed in nuclear extracts derived from mammalian cells. Based on these findings, we searched for endogenous $S$. pombe ESEs downstream from introns that are inherently poor splicing substrates; purine-rich elements identified by this strategy also promote efficient splicing of our reporter constructs. Finally, we show that the essential fission yeast SR protein Srp2p interacts in the three-hybrid system with purine-rich elements from both vertebrates and $S$. pombe; conversely, mammalian SR proteins specifically recognize the putative $S$. pombe ESEs. Taken together, these results provide strong evidence supporting an ancestral role for splicing enhancers. 
Webb et al.

\section{Results \\ Vertebrate exonic splicing enhancers promote splicing of the second intron of cgs2}

The presence of both subunits of U2AF and a highly conserved SR protein does not, a priori, imply the existence of ESEs in $S$. pombe, as these factors play additional roles in splicing and may also function in other aspects of RNA metabolism (Black 2003). However, three observations suggested that these elements are present in fission yeast. First, the integrity of the RS domains on both fission yeast SR proteins is essential for proper function in vivo (Gross et al. 1998; Lutzelberger et al. 1999), as predicted by some models of ESE-mediated splicing. Second, the $\mathrm{U} 2 \mathrm{AF}^{\mathrm{SM}} / \mathrm{SR}$ protein interaction invoked by the protein bridging model for ESE function (Zuo and Maniatis 1996) is conserved in S. pombe (Webb and Wise 2004). Third, the first step of splicing in $S$. pombe is compromised by purine substitutions in 3'-polypyrimidine tracts (Romfo and Wise 1997), as in mammals, where the need for downstream splicing enhancers has been linked to the presence of suboptimal 3'-splice sites (Zuo and Maniatis 1996). These findings, coupled with the degeneracy of intronic splicing signals and prevalence of multi-intronic pre-mRNAs in $S$. pombe, prompted us to ask whether mammalian ESEs could promote splicing of a fission yeast pre-mRNA.

To assay candidate elements for the ability to function as splicing enhancers in S. pombe, we developed minigene reporter constructs expressing pre-mRNAs that are inherently poor splicing substrates. A similar approach has been used to validate putative ESEs identified by computational methods, with test minigenes that mimic "weak" exons that are skipped unless they contain an enhancer (Fairbrother et al. 2002; Zhang and Chasin 2004). As exon skipping is commonly observed in metazoans (Berget 1995; Lopez 1998; Reed 2000; Smith and Valcarcel 2000) but not in S. pombe (Romfo et al. 2000), it was not possible to use strictly analogous reporters. However, in the course of an earlier study, we demonstrated that that the intron located downstream from the 9-nt micro-exon in fission yeast cgs2 is inefficiently spliced (Romfo et al. 2000). Thus, this pre-mRNA provides a natural substrate for analyzing ESE function in $S$. pombe.

We first examined a sequence derived from the ESE of avian sarcoma-leukosis virus (ASLV) (Katz and Skalka 1990; Fu et al. 1991), which was chosen because it has previously been shown to promote efficient splicing of otherwise poor substrates in a heterologous context (Fu et al. 1991). The ASLV element was used to replace endogenous sequences in the third exon of the cgs2-L minigene centered $+50 \mathrm{nt}$ from the 3 '-splice site (Fig. 1A), which is similar to its natural location in the retroviral RNA (Katz and Skalka 1990). The results of low-cycle number semiquantitative RT-PCR demonstrate that the ASLV ESE dramatically increases splicing of cgs2-L (Fig. 1B); nearly identical results were obtained with RNA derived from three independent $S$. pombe transformants.
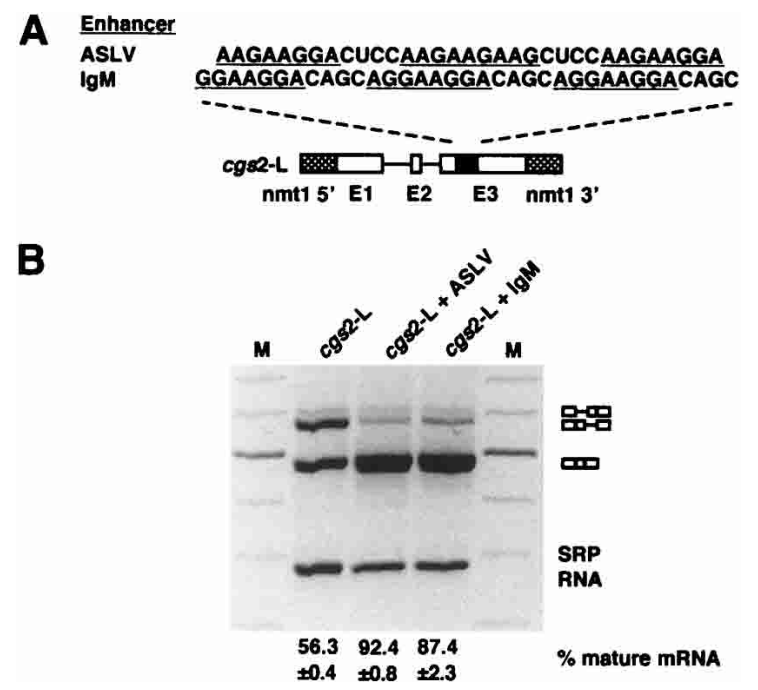

Figure 1. Vertebrate ESEs promote splicing of a micro-exoncontaining pre-mRNA in $S$. pombe. (A) Schematic representation of minigene constructs containing exons 1-3 of $c g s 2$. The ASLV and IgM ESEs replace endogenous cgs2 sequences centered at $+50 \mathrm{nt}$ from the 3 '-splice site of intron 2. (B) RT-PCR splicing assays on total RNA extracted from cells harboring the constructs described in $A$. The identities of $c g s 2$-derived RNA species and the stable cytoplasmic Signal Recognition Particle (SRP) RNA loading control are indicated alongside the gel. Mock RT-PCRs lacking reverse transcriptase indicated no contamination with genomic DNA (data not shown). The signals were quantitated using Bio-Rad Quantity One gel documentation software (version 4.1.1), normalized to RNA length, and the results expressed as percentage precursor accumulation [(precursor RNA)/(precursor mRNAs + mature mRNA) $\times 100]$. Errors indicate standard deviation for three independent splicing assays.

To confirm that the ability to stimulate splicing in fission yeast is a general property of purine-rich vertebrate ESEs, we introduced a different "GAR-type" ESE derived from the mouse immunoglobulin $\mu$ (IgM) gene (Watakabe et al. 1993) at the same position within cgs2-L (Fig. 1A). The effect of this substitution paralleled the robust increase in splicing observed with ASLV (Fig. 1B). As both heterologous substitutions preserve the spacing between the wild-type processing signals in the premRNA, the observed stimulation cannot be due to altering the distance between intron 2 and, for example, the polyadenylation site. We conclude that exonic splicing enhancers that normally function in vertebrate cells can overcome inefficient splicing caused by the presence of a micro-exon in an $S$. pombe pre-mRNA.

Vertebrate ESEs promote splicing of an S. pombe intron containing a suboptimal 3'-splice site

To corroborate and extend our findings with $\operatorname{cgs} 2$, we developed a second series of minigene constructs to test ESE function in $S$. pombe that exploits the effects of weakening the $3^{\prime}$-splice site. We previously demonstrated that replacing the pyrimidine-rich $3^{\prime}$-splice site 
of the second intron of fission yeast $c d c 2$ with purines reduced splicing efficiency, and the effect is even more dramatic if the distance between the branchpoint and 3'-AG is also increased (Romfo and Wise 1997). Thus, these alleles artificially recapitulate the suboptimal 3'splice site naturally found in the Drosophila $d s x$ premRNA, thereby providing a distinct set of poor splicing substrates to evaluate candidate ESEs in S. pombe.

The $c d c 2$ minigenes used are illustrated schematically in Figure 2A, and RT-PCR analysis of splicing is shown in Figure 2B. As shown previously using primer extension analysis (Romfo and Wise 1997), the wild-type allele (cdc2-I2 9Y) is spliced efficiently but not completely, while a polypyrimidine tract replacement with purines that maintains the wild-type branchpoint to 3 '-splice site distance (cdc2-I2 9R) shows diminished splicing, and an 18-purine substitution/insertion ( $c d c 2$-I2 18R) virtually abolishes the mature mRNA signal. Introduction of the ASLV element at $+50 \mathrm{nt}$ from the 3 '-splice site in an otherwise wild-type cdc2-I2 minigene (cdc2-I2 9Y + ASLV) stimulates splicing, as virtually no precursor remains. Similarly, the ASLV enhancer dramatically improves splicing of the all-purine (cdc2-I2 9R + ASLV) allele. In contrast, introduction of the vertebrate ESE into the more severely compromised purine substitution/insertion allele (cdc2-I2 18R + ASLV) produces virtually no increase in splicing. This result supports the argument against one alternative explanation for the results with the $9 \mathrm{Y}$ and $9 \mathrm{R}$ alleles, namely, that instead of promoting

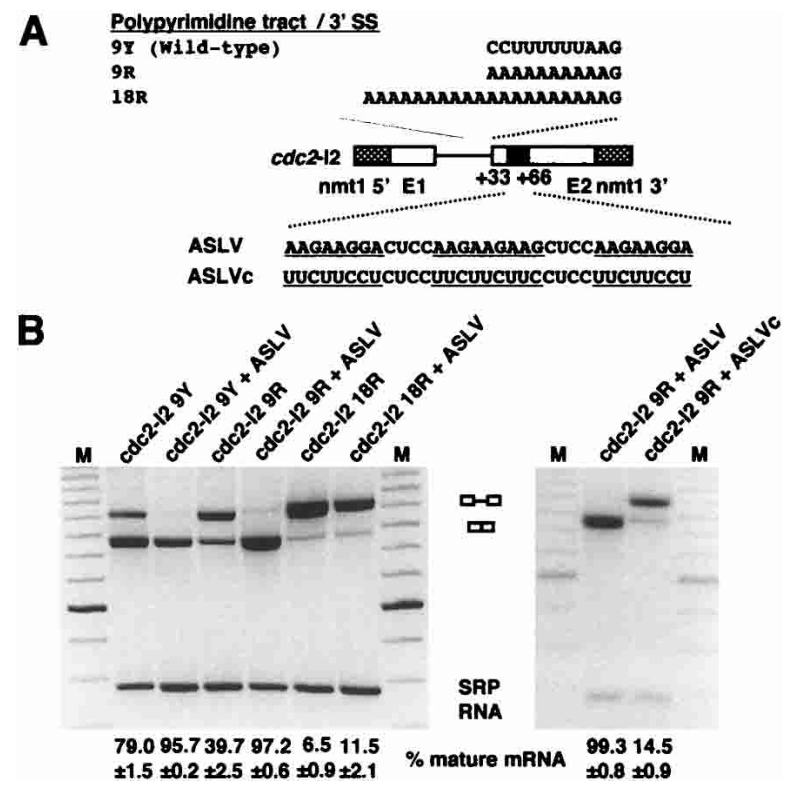

Figure 2. The ASLV ESE promotes splicing of an $S$. pombe pre-mRNA containing a suboptimal 3 '-splice site. (A) Schematic representation of minigene constructs containing $c d c 2$ exons 1-2. The wild-type polypyrimidine tract/3'SS sequence $(9 \mathrm{Y})$, a complete purine replacement $(9 \mathrm{R})$, and a purine replacement/insertion (18R) alleles are shown along with the sequence and position of ASLV and ASLVc. (B) RT-PCR analysis of the constructs depicted in $A$. Splicing assays were performed as in Figure $1 \mathrm{~B}$ except with a $5^{\prime}$-primer specific to $c d c 2$. splicing, the ASLV ESE disrupts a natural splicing silencer (Cartegni et al. 2002). To completely rule out this possibility, we introduced the complement of the ASLV sequence into the $c d c 2$ 9R construct $(c d c 2-\mathrm{I} 29 \mathrm{R}+$ ASLVc). As expected, splicing is not improved.

Our failure to observe increased splicing with the cdc2-I2 18R + ASLV construct further indicates that the increased mRNA levels observed when this ESE was introduced into the other constructs are unlikely to arise via differential stabilization or enhanced nuclear export, as the mature message is identical to those produced from $c d c 2-\mathrm{I} 2$ 9R + ASLV and $c d c 2-\mathrm{I} 29 \mathrm{Y}+$ ASLV. Finally, as for $c g s 2$, introduction of the mouse IgM ESE (Watakabe et al. 1993) into the $c d c 2$ minigenes yields results similar to those observed with ASLV: splicing was stimulated in the 9R but not the $18 \mathrm{R}$ allele (data not shown). We conclude that, as in mammalian cell extracts (Zuo and Maniatis 1996), an ESE can augment the activity of a suboptimal polypyrimidine tract but cannot rescue a completely inactive 3 '-splice site.

\section{ESE activity in fission yeast displays sequence and spacing requirements similar to mammals}

To investigate the extent of ESE functional conservation between $S$. pombe and metazoans, we undertook a series of experiments to test parameters that affect activity in mammalian splicing extracts. In vitro splicing assays have shown that deletions or substitutions of the (GAR) repeats found in ASLV, IgM, and related ESEs diminish or abolish their ability to promote splicing of an upstream intron (Katz and Skalka 1990; Fu et al. 1991; Watakabe et al. 1993; Xu et al. 1993; Dirksen et al. 1994; Tanaka et al. 1994). To assess the dependence of ESE activity in $S$. pombe upon the purine-rich repeats, we constructed two mutant versions of the ASLV ESE located downstream from the 3 '-splice site in the $c d c 2-\mathrm{I} 2$ 9R minigene (Fig. 3A). As illustrated in Figure 3B, replacement of one of three ( $c d c 2-\mathrm{I} 2$ 9R ASLV-1) and two of three (cdc2-I2 9R ASLV-2) of the purine-rich repeats in the vertebrate ESE with native $c d c 2$ exonic sequences dramatically reduces its activity. These data indicate that the purine repeats are responsible for the ability of the ASLV ESE to stimulate splicing in fission yeast, as in extracts from mammalian cells.

In vitro studies of ESE function in mammals have also shown that enhancer activity declines as a function of increased distance from the 3 '-splice site (Lavigueur et al. 1993; Tian and Maniatis 1994; Graveley et al. 1998). To determine whether ESE activity also exhibits distance constraints in fission yeast cells, we constructed two cdc2-I2 9R alleles in which the ASLV element is placed further downstream (Fig. 3C). The first two lanes of the gel shown in Figure 3D are controls reiterating the finding that the ASLV ESE promotes efficient splicing when centered at $+50 \mathrm{nt}$. In contrast, introduction of the ASLV ESE at a position centered $+100 \mathrm{nt}$ downstream of the $3^{\prime}$-splice site has a more modest stimulatory effect, while placement at $+300 \mathrm{nt}$ moves it beyond the effective range, reducing splicing to the baseline level. These 


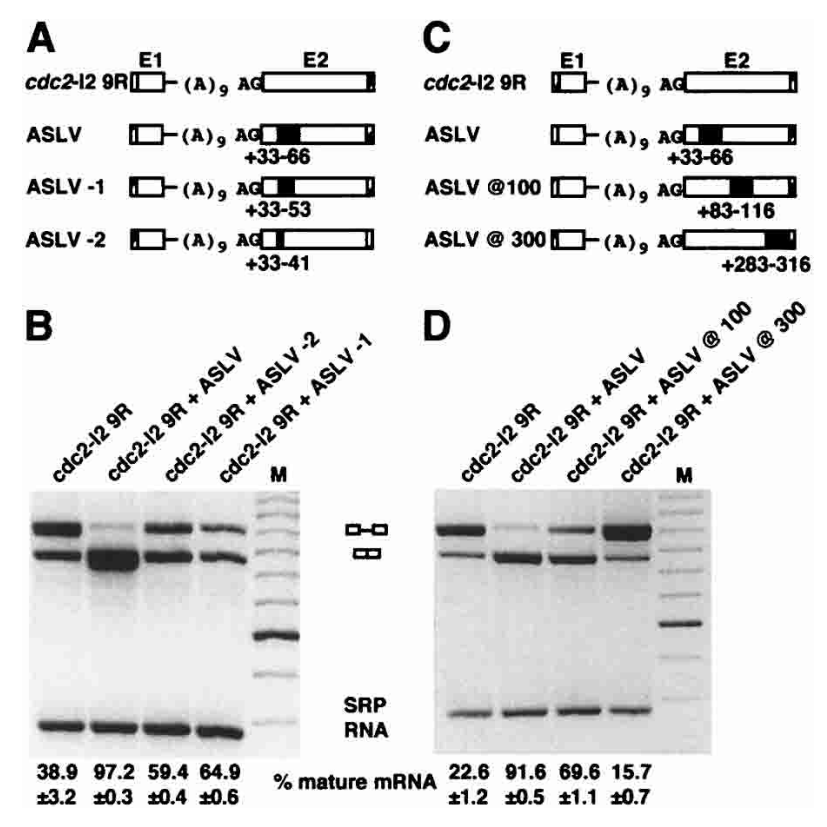

Figure 3. ASLV function in $S$. pombe is affected by changes in sequence and spacing. (A) Schematic representation of $c d c 2-\mathrm{I} 2$ $9 \mathrm{R}$ pre-mRNA variants containing the wild-type and mutant ESEs. (B) Effect of eliminating purine-rich repeats. RT-PCR splicing assays on the constructs described in $A$ were performed as in Figure 2B. (C) Schematic representation of $c d c 2-\mathrm{I} 2$ 9R variants containing ASLV at the indicated locations. (D) Effect of increasing the distance between the ESE and the $3^{\prime}$-splice site of intron 2. RT-PCR splicing assays of the constructs described in $C$ were performed as described in Figure 2B.

results demonstrate that, in $S$. pombe, an ESE functions best when situated $<100 \mathrm{nt}$ distant from the upstream intron. Although a few metazoan ESEs are located $>500$ $\mathrm{nt}$ from their target introns, these are generally complex elements that function in a regulated manner (Hertel et al. 1997). In contrast, constitutive ESEs are generally found within $100 \mathrm{nt}$ of the 3 '-splice site (e.g., Lavigueur et al. 1993; Tian and Maniatis 1993; Xu et al. 1993; Dirksen et al. 1994; Tanaka et al. 1994) and are rendered inactive when moved beyond $300 \mathrm{nt}$ (Tian and Maniatis 1994; Hertel and Maniatis 1998). Thus, the positional requirements for ESE function are conserved between fission yeast and mammals.

A purine-rich element within exon 6 of srp2 $2^{+}$functions as a weak ESE

The ability of mammalian GAR-type ESEs to function in $S$. pombe prompted us to search for endogenous elements that stimulate splicing. We first tested a purinerich sequence found in the sixth exon of $\operatorname{srp} 2^{+}$, which encodes the essential SR protein, Srp2p (Lutzelberger et al. 1999); exon 6 lies downstream from two micro-exons in this complex gene (see Fig. 7A, below). The element, designated SRP2 (Fig. 4A), promotes splicing of the otherwise inefficiently spliced cgs2-L pre-mRNA, which also contains a micro-exon (Fig. 4B). The stimulation was comparable to that observed with ASLV in parallel experiments, and a similar ESE from human cardiac troponin $\mathrm{T}$ (cTNT) (Fig. 4A) was also effective. These results demonstrate that the purine-rich element in the $s r p 2^{+}$gene is similar not only in sequence, but also in function, to mammalian ESEs.

To test whether SRP2 can also promote splicing of an intron containing a suboptimal 3 '-splice site, we introduced it into the $c d c 2-\mathrm{I} 29 \mathrm{R}$ minigene reporter at $+50 \mathrm{nt}$. Unexpectedly, only a modest increase was observed (Fig. $5 \mathrm{~A})$, in contrast to the robust stimulation by ASLV and cTNT. One possible explanation for the discrepancy between the effect of introducing SRP2 into a pre-mRNA harboring a micro-exon versus a suboptimal 3 '-splice site is that they require different modes of splicing activation. In particular, given the similarity between the natural context of SRP2 and cgs2-L, the element might specifically stabilize splicing complexes surrounding micro-exons. Alternatively, SRP2 may be a weak enhancer, and pre-mRNAs with suboptimal 3'-splice sites may have more stringent requirements than those harboring micro-exons.

To distinguish between these possibilities, we constructed $c d c 2-\mathrm{I} 2$ 9R alleles carrying tandem copies of SRP2. Studies in mammalian cell extracts have demonstrated that arrays of ESE sequences increase splicing efficiency in a linear fashion (Hertel and Maniatis 1998). If SRP2 functions specifically in splicing of pre-mRNAs containing micro-exons, then even multiple copies should not activate $c d c 2-\mathrm{I} 2$ 9R splicing. Counter to this prediction, two copies of SRP2 promote splicing of this reporter to the same level observed with a single copy of

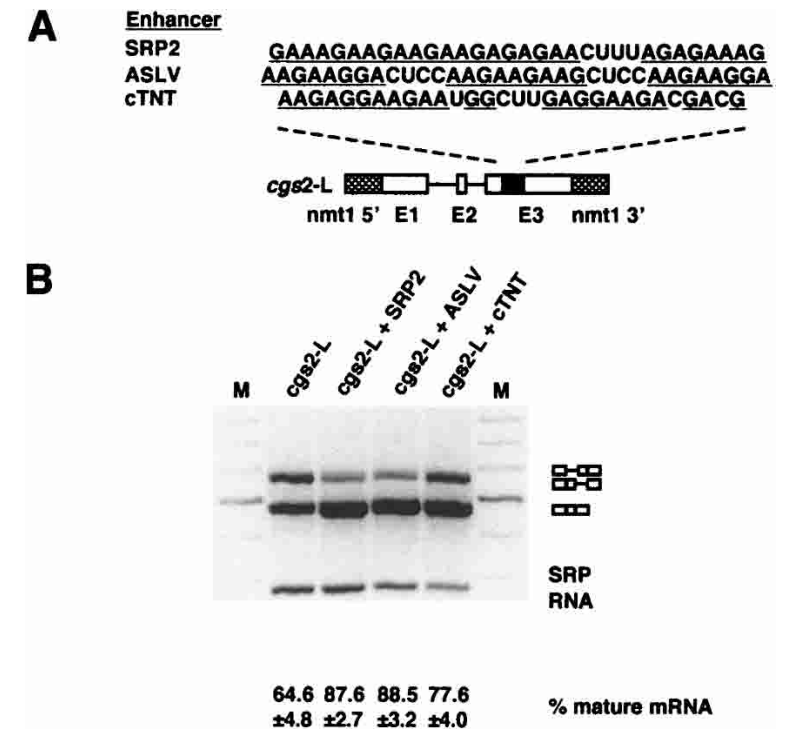

Figure 4. SRP2 promotes splicing of a micro-exon-containing pre-mRNA to the same extent as vertebrate ESEs. $(A)$ Sequences of SRP2, ASLV, and cTNT ESEs and schematic representation of their locations centered at +50 from the 3 '-splice site within cgs2-L. (B) Effect of SRP2, ASLV, and cTNT on cgs2-L splicing. RT-PCR splicing assays of the constructs described in $A$ were performed as in Figure 1B. 
A

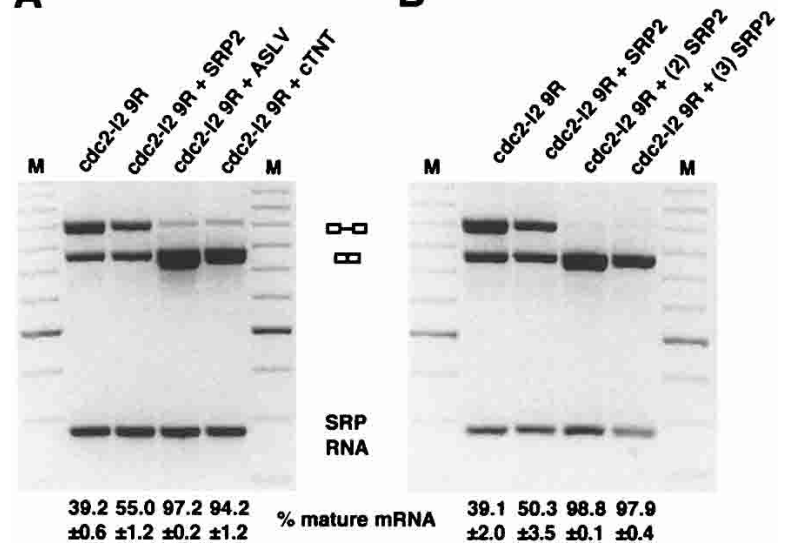

Figure 5. Multiple copies of SRP2 improve splicing of a premRNA containing a suboptimal 3 '-splice site. (A) A single copy of SRP2 does not increase splicing of $c d c 2-\mathrm{I} 29 \mathrm{R}$ to the same extent as the ASLV and cTNT ESEs. RT-PCR splicing assay of $c d c 2-\mathrm{I} 29 \mathrm{R}$ and $c d c 2-\mathrm{I} 29 \mathrm{R}$ variants harboring SRP2, ASLV, and cTNT ESEs centered $+50 \mathrm{nt}$ from the 3 '-splice site, performed as in Figure 2B. (B) Two or three copies of SRP2 promote efficient splicing of $c d c 2-\mathrm{I} 2$ 9R. RT-PCR splicing assays of $c d c 2-\mathrm{I} 2$ 9R and variants harboring single and tandem copies of SRP2 were performed as in Figure 2B. SRP2 substitutions were centered at +50 [cdc2-I2 9R (1) SRP2]; +50, +87 [cdc2-I2 9R (2) SRP2]; +50, $+87,+124[c d c 2-$ I2 9R (3) SRP2] nt from the 3'-splice site.

ASLV or cTNT (Fig. 5, cf. A and B), and three copies are equally effective (Fig. 5B). These results demonstrate that SRP2 is a weak general activator of splicing.

The S. pombe SR protein, Srp2p, interacts with purine-rich ESES

Specific binding of SR proteins is a feature of all models of ESE-mediated splicing activation (Cartegni et al. 2002; Graveley 2004). In contrast to the large mammalian SR protein family with 10 core members (Graveley 2000), the fission yeast genome encodes only two SR proteins (Wood et al. 2002). Srplp is not essential for viability (Gross et al. 1998), while Srp2p is an essential gene (Lutzelberger et al. 1999). To determine whether either or both fission yeast SR proteins can bind SRP2, we used the RNA three-hybrid assay (SenGupta et al. 1996), which has been used in a variety of studies to analyze interactions between splicing factors and RNA (e.g., Matter et al. 2000; Bernstein et al. 2002; Webb and Wise 2004). Notably, Srplp does not interact with the purinerich SRP2, but instead shows a weak interaction with its pyrimidine-rich complement (Fig. 6A). In contrast, Srp2p shows a positive interaction with the element from its own gene, with some growth observed even at $20 \mathrm{mM}$ 3-aminotriazole (3-AT). The Srp2p-SRP2 interaction is specific for purine-rich RNA, as the pyrimidine-rich complement permits growth only to $1 \mathrm{mM}$ (Fig. 6B). We also tested binding of Srp2p to the mammalian GARtype ESEs ASLV and cTNT. Each element interacts strongly, with both strains showing robust growth even at $20 \mathrm{mM}$ 3-AT (Fig. 6B). Again, a purine preference is observed, as RNAs complementary to the ASLV and cTNT ESEs do not allow growth beyond 0.5 and $2.5 \mathrm{mM}$ 3-AT, respectively.

Notably, the results of the three-hybrid assays correlate with our splicing data. In particular, the stronger binding of Srp2p to ASLV and cTNT than to SRP2 correlates with the less robust activity of the latter element in promoting splicing at a suboptimal $3^{\prime}$-splice site (Fig. 5). Similarly, three-hybrid RNA substrates that correspond to the alleles in which ASLV was replaced with endogenous $c d c 2$ sequences (Fig. 3A,B) dramatically reduce Srp2p binding (data not shown). Taken together, these results provide strong evidence that the ability of these purine-rich elements to promote splicing in $S$. pombe is due to their ability to bind Srp2p, as observed for a subset of mammalian SR protein family members (Ramchatesingh et al. 1995).

Because Srp2p is most similar to mammalian ASF/SF2 and SRp55, we tested these SR proteins for binding to the same panel of RNAs. As expected, ASF/SF2 gave strong signals with the purine-rich ASLV and cTNT, but not their pyrimidine-rich complements (Fig. 6C). Binding of ASF/SF2 to SRP2 was not as robust ( $5 \mathrm{mM} 3$-AT) but was still stronger than to its complement $(1 \mathrm{mM})$. Results of $\beta$-galactosidase assays for each of the RNA three-hybrid strains are shown beneath the 3-AT results (Fig. 6D-F). SRp55 displays a similar substrate preference, although the signals were lower than with ASF/SF2 (data not shown). We conclude that mammalian SR proteins are able to recognize an operationally defined fission yeast ESE, providing further evidence for conservation of this mode of splicing activation.

S. pombe GAR-type sequences downstream from micro-exons that are most similar to ASLV promote splicing

Our finding that the SRP2 element, which is found downstream from two micro-exons, functions as a splicing enhancer, combined with our previous demonstration that such pre-mRNAs are inherently inefficient splicing substrates in S. pombe (Romfo et al. 2000), suggested a strategy for identifying additional native splicing enhancers. Based on the hypothesis that ESEs may have evolved and been maintained to ensure the splicing of such pre-mRNAs, we examined a subset of the $\sim 2.5 \%$ of $S$. pombe genes that contain internal micro-exons for downstream purine-rich motifs. In each case, the sequence extending $\sim 100 \mathrm{nt}$ downstream from the 3 '-splice site preceding the exon following the micro-exon was searched by eye for purine-rich islands. Because this nonsystematic search identified five additional candidate elements with similarity to mammalian GAR-type ESEs, we suspect that many more await discovery. Indeed, a recent bioinformatics analysis found matches to mammalian ESEs identified by functional SELEX /Cartegni et al. 2002) in $80 \%$ of the 3159 S. pombe exons examined (Drabenstot et al. 2003); although testing all of these using our minigene constructs is not feasible, we are in the process of identifying a subset for analysis. 
Webb et al.
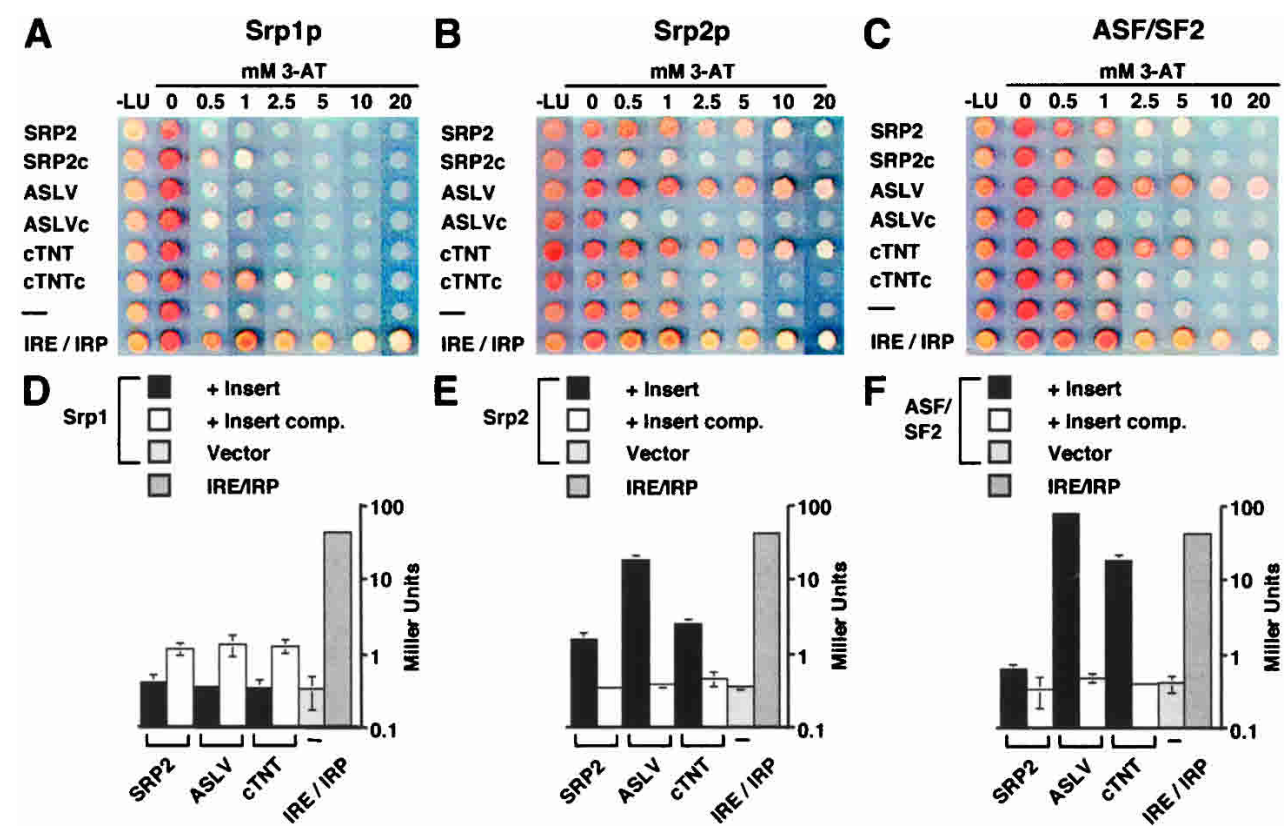

Figure 6. Interactions between purine-rich RNAs and SR proteins are conserved between $S$. pombe and vertebrates. (A) RNA three-hybrid analysis of Srp1p's interaction with SRP2, ASLV, cTNT, and their complementary sequences SRP2c, ASLVc, and cTNTc using a plate assay. The strength of an RNA-protein interaction correlates with growth in the presence of increasing amounts of 3-aminotriazole (3-AT) (SenGupta et al. 1996). Negative (vector) and positive (IRE/IRP) controls are also shown. (B) RNA three-hybrid analysis of the interaction between Srp2p and purine-rich RNA sequences. Assays were performed as in $A$. (C) RNA three-hybrid analysis of the interaction between ASF/SF2 and purine-rich RNA sequences. Assays were performed as in $A$. $(D-F)$ Histograms depicting quantitation of $\beta$-galactosidase assays of the RNA-protein interactions. Results are shown on a logarithmic scale to differentiate the low levels of reporter activation between strains. Error bars indicate standard deviation for three independent assays.

The locations of the newly identified candidate exonic splicing enhancers in their endogenous genes are shown in Figure 7A. We introduced each purine-rich element into the most stringent test minigene (cdc2-I2 9R) and analyzed splicing by RT-PCR. The results (Fig. 7B) demonstrate that four of the five sequences (SPAC12B10.05, trk2, ub11, and SPAC22F3.11c) increase mature mRNA from the baseline level to near completion. In contrast, the element from exon 4 of hus 5 was not able to activate splicing at a suboptimal 3 '-splice site even to the limited extent observed for SRP2. Thus, not all purine-rich sequences can function as ESEs in S. pombe.

The locations of the purine-rich elements within the exon/intron architecture of each micro-exon-containing pre-mRNA provide no obvious clues to their ESE activity in S. pombe: neither distance from the upstream micro-exon nor overall position in the pre-mRNA correlates with the extent to which each stimulates splicing. Similarly uninformative are the demonstrated or inferred functions of each gene product. However, an alignment of the purine-rich elements tested from fission yeast with ASLV demonstrates that the four strong ESEs are more similar to ASLV than either SRP2 or the inactive purine-rich element from hus5 (Fig. 7C). This correlation further supports the hypothesis that ESE-mediated splicing is an evolutionarily ancient mechanism that arose before the divergence of fission yeast and humans.
Mutating the SRP2 element does not have a dramatic effect in its native context

The fact that a high fraction of the candidate ESEs we tested are active in the context of reporter genes begs the question of whether they also promote splicing of the endogenous pre-mRNAs in which they reside. We selected the SRP2 element as the first target to address this question based on the possibility that it might be part of an autoregulatory circuit. To test whether SRP2 is required for srp2 splicing, a portion of the gene was introduced into the nmt1 expression vector, and at the same time, a parallel construct was made in which the putative ESE was replaced with its complement (Fig. 8A). Surprisingly, both pre-mRNAs showed very similar profiles of splicing products (Fig. 8B). Since vertebrate enhancers are believed to promote exon inclusion by mediating communication with downstream 5 '-splice sites (Berget 1995), we tested whether mutating the $5^{\prime}$-splice site abutting exon 6 would produce an effect in combination with mutating the purine-rich element. As shown in Figure 8B, this resulted in a discernible, albeit small, increase in the accumulation of partially spliced intermediates containing one of the two introns just upstream from the putative ESE. This effect was reproducible, and was not observed upon mutating the $5^{\prime}$-splice site alone. Thus, these results raise the possibility that there may be communication across exon 6 in the srp2 
A

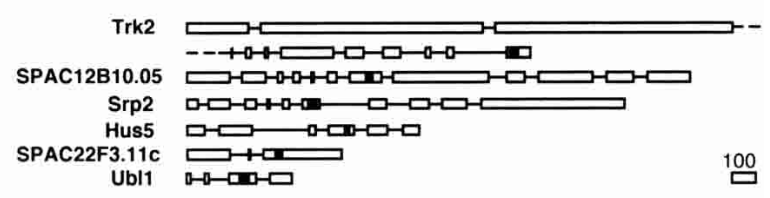

B

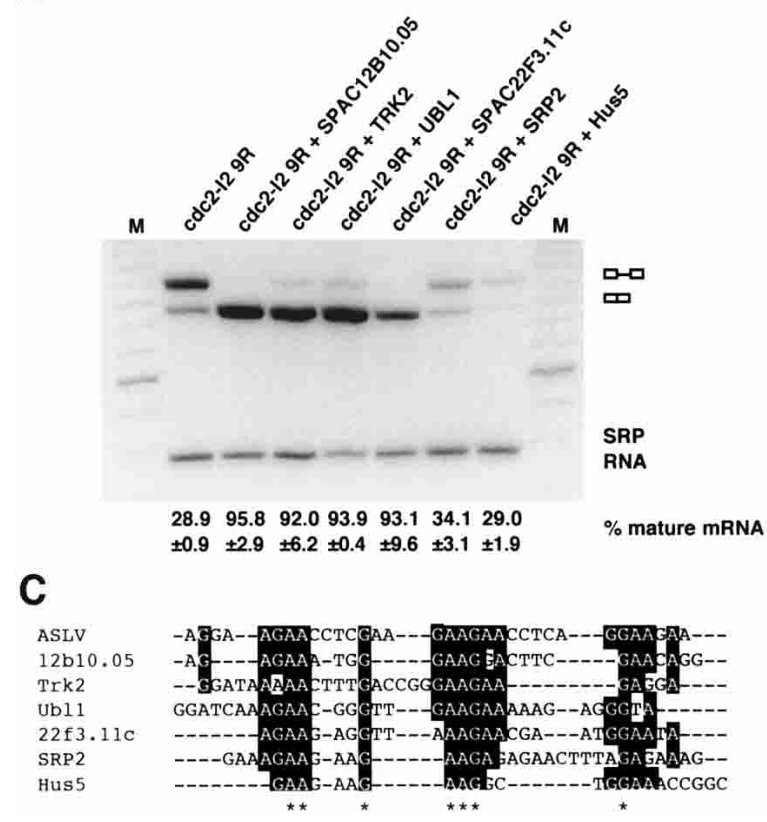

Figure 7. Purine-rich elements derived from other micro-exoncontaining $S$. pombe genes promote splicing of a pre-mRNA containing a suboptimal 3 '-splice site. $(A)$ Schematic representation of exon/intron architecture for $S$. pombe pre-mRNAs harboring purine-rich sequences downstream from micro-exons. Black boxes indicate the position of the elements. (B) Effect of introducing the purine-rich elements at $+50 \mathrm{nt}$ downstream from a suboptimal 3 '-splice site. RT-PCR splicing assays of the cdc2-I2 9R minigene construct and variants harboring the newly identified purine-rich elements, performed as in Figure 2B. (C) Alignment of the fission yeast purine-rich elements with the ASLV ESE using CLUSTALW. Asterisks below the sequences indicate completely conserved positions. Black boxes with white type indicate positions where a purine in the ASLV sequence aligns with an identical nucleotide in all of the putative $S$. pombe ESEs.

pre-mRNA; this is contrary to our earlier proposal that exon definition does not occur in $S$. pombe, based on our inability to induce skipping by mutating the splice sites flanking exons that lack purine-rich sequences (Romfo et al. 2000).

There are several possible explanations for why replacing the putative SRP2 enhancer with its complement did not have more dramatic consequences. First, the enhancer may be critical for splicing under growth conditions that we did not examine or in different phases of life, for example, during meiosis or at a particular stage of the cell cycle. The latter possibility is particularly appealing in light of evidence that SRPK1, a kinase that phosphorylates SR proteins, resides in the cytoplasm ex- cept during metaphase both in fission yeast and humans (Takeuchi and Yanagida 1993; Gui et al. 1994). Second, enhancer function may be bypassed in the heterologous context in which we have examined the mutants. Consistent with this possibility, we recently found that several $S$. pombe pre-mRNAs that are normally spliced only during meiosis are fully matured even during vegetative growth when expressed under nmt1 control (N. Averbeck, J. Leatherwood, S. Sunder, and J.A. Wise, unpubl.). Third, it is possible that the changes we made are not sufficiently extensive; for example, the double-sex enhancer contains purine-rich repeats but these are embedded within a more complex element in the native gene (Lynch and Maniatis 1995). Notably, the purine-rich region of dsx, like the SRP2 element, can function as a constitutive splicing enhancer when placed in a heterologous context (Zuo and Maniatis 1996). Our future work will be directed toward testing these hypotheses regarding SRP2 enhancer function, as well as examining the other candidate enhancers in their native contexts.

\section{Discussion}

This study presents the first experimental demonstration that GAR-type ESEs promote splicing in a unicellular eukaryote and provides evidence that they do so by a conserved mechanism. In strong support of the idea that

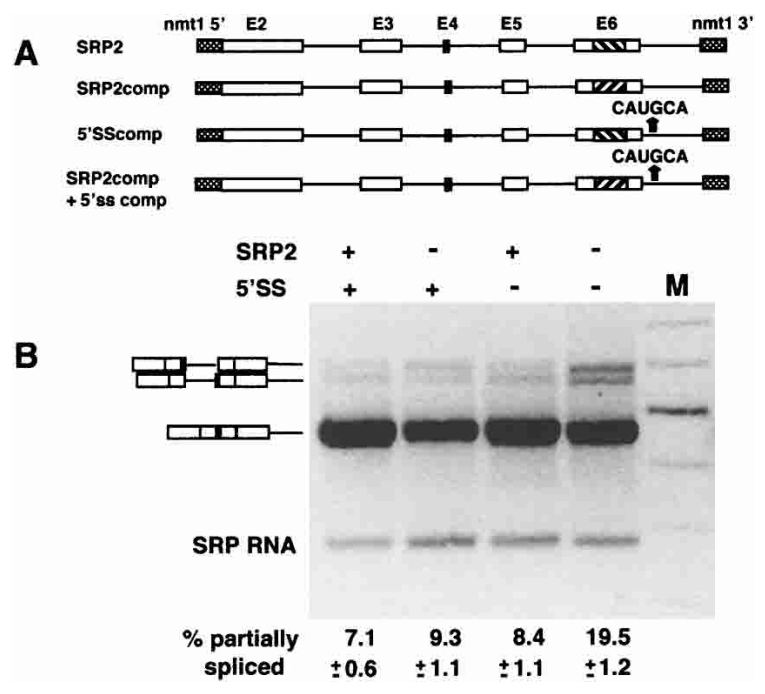

Figure 8. Mutating the putative ESE in an srp2 minigene construct diminishes splicing only in combination with a mutant downstream 5'-splice site. (A) Schematic representation of the wild-type and mutant srp2 pre-mRNAs carrying either the complement of the SRP2 purine-rich element (reverse hatching), the complement of the downstream $5^{\prime}$-splice site, or both. The constructs included exons 2-6 inserted into the nmt1 expression cassette. The 6-nt micro-exon is indicated in black. $(B)$ RT-PCR analysis of the wild-type and mutant srp2 pre-mRNAs produced from the minigenes. Splicing assays were performed as in Figure 1B except with a $5^{\prime}$-primer specific to srp2 exon 2. Constructs that contain the wild-type purine-rich element or 5 '-splice site are denoted by a plus sign $(+)$ above the gel, while a minus sign (-) indicates the presence of the complementary sequence. 
exonic enhancers arose early in eukaryotic evolution, we find that each mammalian ESE tested promotes splicing of two distinct fission yeast pre-mRNAs, and, conversely, mammalian SR proteins bind specifically to a purine-rich exonic element from $S$. pombe that functions as a splicing enhancer.

\section{ESE-mediated splicing is ancient but not ubiquitous}

Since their radiation from a common ancestor, S. cerevisiae has eliminated several groups of functionally linked genes, including some components of the spliceosome, which are preserved in S. pombe (Aravind et al. 2000). Among the lost genes are those encoding factors implicated in ESE-mediated splicing (Aravind et al. 2000; Kaufer and Potashkin 2000). Although a randomization/ functional selection strategy in budding yeast did elicit 5 '-splice site variants, these were not tested in exonic locations (Libri et al. 2000), and natural examples of splicing enhancement in $S$. cerevisiae are mediated by intronic sequences. These either function in constitutive splicing to juxtapose the 5 '-splice site and branchpoint via base pairing (Libri et al. 1995) or serve as proteinbinding sites to mediate positive regulation during meiosis (Spingola and Ares 2000). In contrast, the machinery that recognizes and responds to exonic splicing enhancers is conserved in $S$. pombe, and we have now provided evidence that the cognate cis-acting signals are operational.

\section{Evidence for conservation of the ESE mechanism in $\mathrm{S}$. pombe}

Several pieces of data presented here and elsewhere provide evidence that exonic splicing enhancers function by similar mechanisms in fission yeast and metazoans. First, the results of mutating the purine-rich repeats in the ASLV element or altering its distance from the target intron suggest similar requirements in cis. Second, multiple copies of a weak ESE increase splicing efficiency beyond the level observed with a single copy, as in mammalian splicing extracts (Hertel and Maniatis 1998; Zhang and Chasin 2004). SR proteins are the trans-acting factors that recognize purine-rich ESEs in both $S$. pombe and vertebrates. Our protein-binding data are compatible with both the original U2AF recruitment model (Wang et al. 1995; Zuo and Maniatis 1996) and the recently proposed facilitated U2 snRNA annealing model (Shen et al. 2004b) of exonic enhancer function, as the cornerstone in each case is binding of one or more SR proteins to the element. Mammalian SR proteins contact ESEs via their RRM domains, and mutating RNP1 of fission yeast Srp2p abolishes binding to purine-rich elements in the three-hybrid system (C.J. Webb and J.A. Wise, unpubl.), providing an additional parallel.

In support of the idea that U2AF recruitment is relevant to ESE function in $S$. pombe, we recently reported that Srp2p interacts specifically with the small subunit of U2AF (Webb and Wise 2004). Interestingly, Srplp did not interact with $\mathrm{U}_{2} \mathrm{AF}^{\mathrm{SM}}$ in our earlier study and also does not bind to purine-rich ESEs in the present study. Taken together, these results suggest that the essential Srp2 protein is the central player in mediating enhancer function in fission yeast. While a clear role for the nonessential Srplp has yet to emerge, it may be relevant that it interacts with Srp2p both in the two-hybrid system and in GST pull-downs (Tang et al. 2002). Thus, Srplp may serve to augment or inhibit Srp2p function, as has been proposed for mammalian SR proteins ( Wu and Maniatis 1993).

\section{What is the role of ESEs in S. pombe splicing?}

In metazoans, ESEs are a component of the alternative splicing engine that drives both proteome diversity and its temporal and spatial regulation (Maniatis and Tasic 2002). While the array of protein variants made possible by differential splicing offsets the lower than predicted number of genes in the human genome, thereby closing the "complexity gap" (Lareau et al. 2004), the numbers of genes and proteins are likely to more closely match in $S$. pombe. In particular, the predominant $(63 \%)$ mode of alternative splicing in humans (Clark and Thanaraj 2002) is skipping of "cassette" exons, as predicted by the exon definition model (Berget 1995), whereas in S. pombe, both exon/intron architecture and mutational analysis suggest that splice sites are generally paired via intron definition (Berget 1995; Romfo et al. 2000). Indeed, no natural examples of exon skipping have been reported in $S$. pombe, and it is difficult to induce experimentally (Romfo et al. 2000).

If exonic splicing enhancers do not generally participate in cross-exon interactions in fission yeast, as appears to be the case in mammals (Lam and Hertel 2002), what do they do? We propose that the primary function of these elements in $S$. pombe is to recruit the splicing machinery to upstream introns that would otherwise be inefficiently spliced due to splicing signals that deviate from the consensus sequences, upstream micro-exons, or other as-yet-unknown reasons. Consistent with a function in constitutive splicing, binding motifs for the mammalian proteins most closely related to Srp2p were most common among the several thousand sequences that resemble mammalian ESEs found by bioinformatics analysis of $S$. pombe exons (Drabenstot et al. 2003). The broad distribution of ESE-related elements in $S$. pombe, coupled with our demonstration that both native and heterologous purine-rich elements dramatically improve splicing of two poor splicing substrates when positioned close to the 3 '-splice site, points to a general role in splicing. Thus, as in mammals, the additional information provided by exonic enhancers may be necessary for the $S$. pombe genome to maintain a large number of introns with highly degenerate splicing signals. Conversely, while distinguishing between evolutionary cause and effect is difficult, the parallel loss of introns and factors implicated in ESE function in S. cerevisiae seems likely to reflect a relief of selective pressure. 
Regulated/differential splicing in S. pombe?

While it is possible that fission yeast ESEs function exclusively in constitutive splicing, a remarkable number of genes in this organism display complex exon/intron architecture: for example, there are 326 genes with four or more exons and 154 with five or more (Wood et al. 2002). Among this group is $\operatorname{srp} 2^{+}$, which contains $10 \mathrm{ex}-$ ons (Fig. 7A). In addition to its complexity, the presence of a purine-rich element that binds the product of this gene makes Srp2p an excellent candidate to regulate its own expression. However, our initial effort to unlock the mysteries of the SRP2 enhancer (Fig. 8) leave many questions unanswered, and these are being investigated in current studies. Consistent with our hypothesis, autoregulation at the level of splicing has been reported to control the levels of both canonical SR proteins (Jumaa and Nielsen 1997; Lopato et al. 1999; Lejeune et al. 2001) and SR-related factors (Stoilov et al. 2004) in other organisms.

As noted above, natural instances of exon skipping have not yet been found in fission yeast, yet it is this form of alternative splicing that is most closely associated with ESEs in metazoans. However, there is another paradigm for how purine-rich elements such as those identified here might regulate splicing in S. pombe. Precedent is provided by pre-mRNAs from vertebrates and their viruses that use exonic splicing enhancers to modulate intron retention or removal (e.g., Hampson et al. 1989; Katz and Skalka 1990). The consequences of retention can be either production of an alternative form of the protein if the intron encodes a continuous open reading frame, or a truncated protein if an in-frame stop codon is present (e.g., Hampson et al. 1989; Jumaa and Nielsen 1997).

Two potential cases of differential splicing via retention of introns with continuous open reading frames have been reported in S. pombe; interestingly, one of these encodes a splicing factor and the other a transcription factor, and both the partially and fully spliced products are predicted to produce functional proteins (Habara et al. 1998; Okazaki and Niwa 2000). In addition, more than a dozen pre-mRNAs that display intron retention except during meiosis have recently come to light $(\mathrm{N}$. Averbeck and J. Leatherwood, pers. comm.). Given the limited analysis of splicing in this organism, it is reasonable to predict that other examples of differential or regulated splicing remain to be discovered. As both the cisacting elements and trans-acting factors necessary for ESE-mediated control of intron retention are conserved in $S$. pombe, we hypothesize that these contribute to mechanisms for increasing proteomic diversity, perhaps helping to explain why the fission yeast genome contains substantially fewer genes than that of baker's yeast.

\section{Materials and methods}

\section{Plasmid construction and mutagenesis}

Minigene constructs were generated by either site-directed mutagenesis (Amersham Corp.) on single-stranded DNA or overlap extension PCR (Romfo et al. 2000) with Platinum $p f_{X}$ DNA polymerase (Invitrogen Corp.). Mutagenesis of the third exon of cgs2 to replace endogenous sequences with ASLV and IgM used single-stranded cgs2-WT/pREP1 template (Romfo et al. 2000) and oligonucleotides 1 and 2 (Supplementary Table 1). Polypyrimidine tract mutants in $c d c 2-\mathrm{I} 2 / \mathrm{pREP} 2$ were described previously (Romfo and Wise 1997); the alleles used here differ slightly in that they contain full-length exon 3. A PCR fragment encoding the remainder ( $235 \mathrm{nt}$ ) of the third exon was generated using oligonucleotides 9 and 10 and inserted into the BamHI sites of wild-type, 9R, and 18R alleles. Replacement of exon 3 sequences with vertebrate ESEs was carried out with oligonucleotides 3 and 4 . Oligonucleotides 5 and 6 were used to replace ASLV sequences with endogenous $c d c 2$ sequence. To move the ASLV ESE farther downstream from the 3 '-splice site of $c d c 2$-I2, we used oligonucleotides 7 and 8.

All other minigenes were produced by overlap extension PCR: $c d c 2-\mathrm{I} 2$ 9R + ASLVc was generated with the external oligonucleotides 13 and 14 and the internal oligonucleotides 15 and 17 with cdc2-I2 9R/pREP2 as template; cgs2-L + SRP2 and cgs $2-\mathrm{L}+\mathrm{cTNT}$ were made with the internal oligonucleotides 19, 20, and 21 and the external oligonucleotides cgs2int1-5' and cgs2int1-3' using cgs2-WT/pREP1 as template; $c d c 2-\mathrm{I} 2$ $9 \mathrm{R}+\mathrm{SRP} 2$ and $c d c 2-\mathrm{I} 29 \mathrm{R}+\mathrm{cTNT}$ were made with the internal oligonucleotides 16,17, and 18 and the external oligonucleotides 13 and 14 with $c d c 2-\mathrm{I} 2$ 9R/pREP2 as template; $c d c 2-\mathrm{I} 2$ 9R + (2) SRP2 and cdc2-I2 9R + (3) SRP2 were made with the internal oligonucleotides 22,23 , and 24 and the external oligonucleotides 13 and 14 with the $c d c 2-\mathrm{I} 29 \mathrm{R}+\mathrm{spE} / \mathrm{pREP} 2$ template. The candidate $S$. pombe ESE cdc2-I2 9R series (Trk2, Hus5, Ubl1, SPAC12B10.05, SPAC22F3.11c) were made with internal oligonucleotides 17 and 25-29, respectively, with the external oligonucleotides 13 and 14 and $c d c 2-I 2$ 9R/pREP2 template. The srp2 minigene constructs were made with internal oligonucleotides 45 (both enhancer and 5' splice site mutated to complement) and 46 (only enhancer mutated to complement) in combination with the external oligonucleotides 42 and 43.

The Srp1p/pACT2 plasmid was prepared by RT-PCR amplification of total RNA with oligonucleotides 11 and 12 and subcloning into pACT2 (BD Sciences Clontech). Oligonucleotides 30-41 were 5'-phosphorylated, annealed, and inserted into the XmaI and ClaI sites of p4130 (described in Fewell and Woolford 1999) (kindly provided by J. Woolford, Carnegie Mellon University, Pittsburgh, PA) to generate SRP2; SRP2c; ASLV; ASLVc; cTNT and cTNTc/pIII MS2-1B, H, and C for use in the RNA three-hybrid system.

\section{RNA preparation and analysis}

The S. pombe strain DS-2 ( $\mathrm{h}^{+}$, ade6-M210, leu1-32, ura4-d18) was the host for assaying splicing of the $c d c 2$ and $c g s 2$ variant minigenes (Romfo and Wise 1997). Preparation of RNA and RTPCR splicing assays were performed as previously described (Webb and Wise 2004). Analysis of $c d c 2$ variant constructs was performed with oligonucleotides 13 and 47; the cgs2 variant construct splicing was assayed with cgs2int1-5' and oligonucleotide 47; the srp2 variant construct splicing was assayed with oligonucleotides 42 and 47 . Levels of the SRP RNA loading control were assayed with oligonucleotides $48-50$. For each construct described, RT-PCR splicing assays were performed in triplicate for at least two independent transformants.

\section{RNA three-hybrid analysis}

Plasmids encoding the activation domain fused to Srplp (described above), Srp2p, and ASF/SF2 (Webb and Wise 2004) were 
cotransformed with the RNA expression pIII MS2-1B, H, C vector series (described above) into the L40 $\mathrm{S}$. cerevisiae

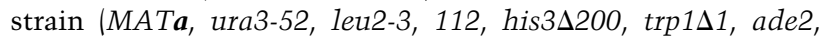
LYS2::(lexAop)-HIS3, ura3::(lexAop)-lacZ; kindly provided by J. Woolford) (SenGupta et al. 1996). Duplicate transformants were grown to mid-log phase and replica-plated onto media containing various amounts 3 -AT. $\beta$-Galactosidase assays were performed in triplicate (Webb and Wise 2004).

\section{Acknowledgments}

We wish to express our gratitude to Hua Lou and David McPheeters for critical comments on the manuscript. We thank Brett Garson for technical assistance and the curators of the Sanger Center $S$. pombe genome project for providing a list of genes containing micro-exons. This research was supported by a grant to J.A.W. from the National Institutes of Health; C.J.W. was supported in part through a Cell and Molecular Biology Training Grant awarded through NIGMS.

\section{References}

Aravind, L., Watanabe, H., Lipman, D.J., and Koonin, E.V. 2000. Lineage-specific loss and divergence of functionally linked genes in eukaryotes. Proc. Nat1. Acad. Sci. 97: 11319-11324.

Aznarez, I., Chan, E.M., Zielenski, J., Blencowe, B.J., and Tsui, L.C. 2003. Characterization of disease-associated mutations affecting an exonic splicing enhancer and two cryptic splice sites in exon 13 of the cystic fibrosis transmembrane conductance regulator gene. Hum. Mol. Genet. 12: 2031-2040.

Berget, S.M. 1995. Exon recognition in vertebrate splicing. J. Biol. Chem. 270: 2411-2414.

Bernstein, D.S., Buter, N., Stumpf, C., and Wickens, M. 2002. Analyzing mRNA-protein complexes using a yeast threehybrid system. Methods 26: 123-141.

Black, D.L. 2003. Mechanisms of alternative pre-messenger RNA splicing. Annu. Rev. Biochem. 72: 291-336.

Caputi, M. and Zahler, A.M. 2002. SR proteins and hnRNP H regulate the splicing of the HIV-1 tev-specific exon 6D. $E M B O$ J. 21: 845-855.

Caputi, M., Kendzior Jr., R.J., and Beemon, K.L. 2002. A nonsense mutation in the fibrillin-1 gene of a Marfan syndrome patient induces NMD and disrupts an exonic splicing enhancer. Genes \& Dev. 16: 1754-1759.

Cartegni, L. and Krainer, A.R. 2002. Disruption of an SF2/ASFdependent exonic splicing enhancer in SMN2 causes spinal muscular atrophy in the absence of SMN1. Nat. Genet. 30: 377-384.

Cartegni, L., Chew, S.L., and Krainer, A.R. 2002. Listening to silence and understanding nonsense: Exonic mutations that affect splicing. Nat. Rev. Genet. 3: 285-298.

Clark, F. and Thanaraj, T.A. 2002. Categorization and characterization of transcript-confirmed constitutively and alternatively spliced introns and exons from human. Hum. Mol. Genet. 11: 451-464.

Coulter, L.R., Landree, M.A., and Cooper, T.A. 1997. Identification of a new class of exonic splicing enhancers by in vivo selection. Mol. Cell. Biol. 17: 2143-2150.

Dirksen, W.P., Hampson, R.K., Sun, Q., and Rottman, F.M. 1994. A purine-rich exon sequence enhances alternative splicing of bovine growth hormone pre-mRNA. I. Biol. Chem. 269: 6431-6436.

Drabenstot, S.D., Kupfer, D.M., White, J.D., Dyer, D.W., Roe, B.A., Buchanan, K.L., and Murphy, J.W. 2003. FELINES: A utility for extracting and examining EST-defined introns and exons. Nucleic Acids Res. 31: e141.

Fairbrother, W.G., Yeh, R.F., Sharp, P.A., and Burge, C.B. 2002. Predictive identification of exonic splicing enhancers in human genes. Science 297: 1007-1013.

Fedorov, A., Saxonov, S., Fedorova, L., and Daizadeh, I. 2001. Comparison of intron-containing and intron-lacking human genes elucidates putative exonic splicing enhancers. Nucleic Acids Res. 29: 1464-1469.

Fewell, S.W. and Woolford Jr., J.L. 1999. Ribosomal protein S14 of Saccharomyces cerevisiae regulates its expression by binding to RPS14B pre-mRNA and to 18S rRNA. Mol. Cell. Biol. 19: 826-834.

Fu, X.D., Katz, R.A., Skalka, A.M., and Maniatis, T. 1991. The role of branchpoint and 3 '-exon sequences in the control of balanced splicing of avian retrovirus RNA. Genes \& Dev. 5: 211-220.

Galiana-Arnoux, D., Lejeune, F., Gesnel, M.C., Stevenin, J., Breathnach, R., and Del Gatto-Konczak, F. 2003. The CD44 alternative $\mathrm{v} 9$ exon contains a splicing enhancer responsive to the SR proteins 9G8, ASF/SF2, and SRp20. J. Biol. Chem. 278: 32943-32953.

Graveley, B.R. 2000. Sorting out the complexity of SR protein functions. RNA 6: 1197-1211.

-2004. A protein interaction domain contacts RNA in the prespliceosome. Mol. Cell 13: 302-304.

Graveley, B.R. and Maniatis, T. 1998. Arginine/serine-rich domains of SR proteins can function as activators of premRNA splicing. Mol. Cell 1: 765-771.

Graveley, B.R., Hertel, K.J., and Maniatis, T. 1998. A systematic analysis of the factors that determine the strength of premRNA splicing enhancers. EMBO I. 17: 6747-6756.

. 2001. The role of U2AF35 and U2AF65 in enhancerdependent splicing. RNA 7: 806-818.

Gross, T., Richert, K., Mierke, C., Lutzelberger, M., and Kaufer, N.F. 1998. Identification and characterization of srp1, a gene of fission yeast encoding a RNA binding domain and a RS domain typical of SR splicing factors. Nucleic Acids Res. 26: 505511.

Gui, J.F., Lane, W.S., and Fu, X.D. 1994. A serine kinase regulates intracellular localization of splicing factors in the cell cycle. Nature 369: 678-682.

Guth, S., Martinez, C., Gaur, R.K., and Valcarcel, J. 1999. Evidence for substrate-specific requirement of the splicing factor $\mathrm{U} 2 \mathrm{AF}(35)$ and for its function after polypyrimidine tract recognition by U2AF(65). Mol. Cell. Biol. 19: 8263-8271.

Guth, S., Tange, T.O., Kellenberger, E., and Valcarcel, J. 2001. Dual function for U2AF(35) in AG-dependent pre-mRNA splicing. Mol. Cell. Biol. 21: 7673-7681.

Habara, Y., Urushiyama, S., Tani, T., and Ohshima, Y. 1998. The fission yeast prp $10^{+}$gene involved in pre-mRNA splicing encodes a homologue of highly conserved splicing factor, SAP155. Nucleic Acids Res. 26: 5662-5669.

Hampson, R.K., La Follette, L., and Rottman, F.M. 1989. Alternative processing of bovine growth hormone mRNA is influenced by downstream exon sequences. Mol. Cell. Biol. 9: 1604-1610.

Hastings, M.L. and Krainer, A.R. 2001. Pre-mRNA splicing in the new millennium. Curr. Opin. Cell Biol. 13: 302-309.

Hertel, K.J. and Maniatis, T. 1998. The function of multisite splicing enhancers. Mol. Cell 1: 449-455.

Hertel, K.J., Lynch, K.W., and Maniatis, T. 1997. Common themes in the function of transcription and splicing enhancers. Curr. Opin. Cell Biol. 9: 350-357.

Hoffman, B.E. and Grabowski, P.J. 1992. U1 snRNP targets an essential splicing factor, U2AF65, to the 3 ' splice site by a 
network of interactions spanning the exon. Genes \& Dev. 6: $2554-2568$.

Jumaa, H. and Nielsen, P.J. 1997. The splicing factor SRp20 modifies splicing of its own mRNA and ASF/SF2 antagonizes this regulation. EMBO J. 16: 5077-5085.

Kan, J.L. and Green, M.R. 1999. Pre-mRNA splicing of IgM exons M1 and M2 is directed by a juxtaposed splicing enhancer and inhibitor. Genes \& Dev. 13: 462-471.

Katz, R.A. and Skalka, A.M. 1990. Control of retroviral RNA splicing through maintenance of suboptimal processing signals. Mol. Cell. Biol. 10: 696-704.

Kaufer, N.F. and Potashkin, J. 2000. Analysis of the splicing machinery in fission yeast: A comparison with budding yeast and mammals. Nucleic Acids Res. 28: 3003-3010.

Kuhn, A.N. and Kaufer, N.F. 2003. Pre-mRNA splicing in Schizosaccharomyces pombe: Regulatory role of a kinase conserved from fission yeast to mammals. Curr. Genet. 42: 241-251.

Lam, B.J. and Hertel, K.J. 2002. A general role for splicing enhancers in exon definition. RNA 8: 1233-1241.

Lareau, L.F., Green, R.E., Bhatnagar, R.S., and Brenner, S.E. 2004. The evolving roles of alternative splicing. Curr. Opin. Struct. Biol. 14: 273-282.

Lavigueur, A., La Branche, H., Kornblihtt, A.R., and Chabot, B. 1993. A splicing enhancer in the human fibronectin alternate ED1 exon interacts with SR proteins and stimulates U2 snRNP binding. Genes \& Dev. 7: 2405-2417.

Lejeune, F., Cavaloc, Y., and Stevenin, J. 2001. Alternative splicing of intron 3 of the serine/arginine-rich protein 9G8 gene. Identification of flanking exonic splicing enhancers and involvement of $9 \mathrm{G} 8$ as a trans-acting factor. J. Biol. Chem. 276: 7850-7858.

Li, Y. and Blencowe, B.J. 1999. Distinct factor requirements for exonic splicing enhancer function and binding of U2AF to the polypyrimidine tract. J. Biol. Chem. 274: 3507435079.

Libri, D., Stutz, F., McCarthy, T., and Rosbash, M. 1995. RNA structural patterns and splicing: Molecular basis for an RNAbased enhancer. RNA 1: 425-436.

Libri, D., Lescure, A., and Rosbash, M. 2000. Splicing enhancement in the yeast rp51b intron. RNA 6:352-368.

Liu, H.X., Zhang, M., and Krainer, A.R. 1998. Identification of functional exonic splicing enhancer motifs recognized by individual SR proteins. Genes \& Dev. 12: 1998-2012.

Lopato, S., Kalyna, M., Dorner, S., Kobayashi, R., Krainer, A.R., and Barta, A. 1999. atSRp30, one of two SF2/ASF-like proteins from Arabidopsis thaliana, regulates splicing of specific plant genes. Genes \& Dev. 13: 987-1001.

Lopez, A.J. 1998. Alternative splicing of pre-mRNA: Developmental consequences and mechanisms of regulation. Annu. Rev. Genet. 32: 279-305.

Lorson, C.L. and Androphy, E.J. 2000. An exonic enhancer is required for inclusion of an essential exon in the SMA-determining gene SMN. Hum. Mol. Genet. 9: 259-265.

Lutzelberger, M., Gross, T., and Kaufer, N.F. 1999. Srp2, an SR protein family member of fission yeast: In vivo characterization of its modular domains. Nucleic Acids Res. 27: 26182626.

Lynch, K.W. and Maniatis, T. 1995. Synergistic interactions between two distinct elements of a regulated splicing enhancer. Genes \& Dev. 9: 284-293.

- 1996. Assembly of specific SR protein complexes on distinct regulatory elements of the Drosophila doublesex splicing enhancer. Genes \& Dev. 10: 2089-2101.

Maniatis, T. and Tasic, B. 2002. Alternative pre-mRNA splicing and proteome expansion in metazoans. Nature 418: 236-243.
Matter, N., Marx, M., Weg-Remers, S., Ponta, H., Herrlich, P., and Konig, H. 2000. Heterogeneous ribonucleoprotein A1 is part of an exon-specific splice-silencing complex controlled by oncogenic signaling pathways. J. Biol. Chem. 275: 3535335360.

Mayeda, A., Screaton, G.R., Chandler, S.D., Fu, X.D., and Krainer, A.R. 1999. Substrate specificities of SR proteins in constitutive splicing are determined by their RNA recognition motifs and composite pre-mRNA exonic elements. Mol. Cell Biol. 19: 1853-1863.

Nelson, K.K and Green, M.R. 1988. Splice site selection and ribonucleoprotein complex assembly during in vitro premRNA splicing. Genes \& Dev. 2: 319-329.

Okazaki, K. and Niwa, O. 2000. mRNAs encoding zinc finger protein isoforms are expressed by alternative splicing of an in-frame intron in fission yeast. DNA Res. 7: 27-30.

Patterson, B. and Guthrie, C. 1991. A U-rich tract enhances usage of an alternative 3' splice site in yeast. Cell 64: 181-187.

Potashkin, J., Naik, K., and Wentz-Hunter, K. 1993. U2AF homolog required for splicing in vivo. Science 262: 573 575.

Ramchatesingh, J., Zahler, A.M., Neugebauer, K.M., Roth, M.B., and Cooper, T.A. 1995. A subset of SR proteins activates splicing of the cardiac troponin $\mathrm{T}$ alternative exon by direct interactions with an exonic enhancer. Mol. Cell. Biol. 15: 4898-4907.

Reed, R. 1989. The organization of 3 ' splice-site sequences in mammalian introns. Genes \& Dev. 3: 2113-2123.

- 2000. Mechanisms of fidelity in pre-mRNA splicing. Curr. Opin. Cell Biol. 12: 340-345.

Reed, R. and Maniatis, T. 1986. A role for exon sequences and splice-site proximity in splice-site selection. Cell 46: 681-690.

Romfo, C.M. and Wise, J.A. 1997. Both the polypyrimidine tract and the $3^{\prime}$ splice site function prior to the first step of splicing in fission yeast. Nucleic Acids Res. 25: 4658-4665.

Romfo, C.M., Lakhe-Reddy, S., and Wise, J.A. 1999. Molecular genetic analysis of U2AF59 in Schizosaccharomyces pombe: Differential sensitivity of introns to mutational inactivation. RNA 5: 49-65.

Romfo, C.M., Alvarez, C.J., van Heeckeren, W.J., Webb, C.J., and Wise, J.A. 2000. Evidence for splice site pairing via intron definition in Schizosaccharomyces pombe. Mol. Cell. Biol. 20: 7955-7970.

Ryner, L.C. and Baker, B.S. 1991. Regulation of doublesex premRNA processing occurs by 3 '-splice site activation. Genes \& Dev. 5: 2071-2085.

Schaal, T.D. and Maniatis, T. 1999. Multiple distinct splicing enhancers in the protein-coding sequences of a constitutively spliced pre-mRNA. Mol. Cell. Biol. 19: 261-273.

Screaton, G.R., Caceres, J.F., Mayeda, A., Bell, M.V., Plebanski, M., Jackson, D.G., Bell, J.I., and Krainer, A.R. 1995. Identification and characterization of three members of the human SR family of pre-mRNA splicing factors. EMBO J. 14: 43364349.

SenGupta, D.J., Zhang, B., Kraemer, B., Pochart, P., Fields, S., and Wickens, M. 1996. A three-hybrid system to detect RNA-protein interactions in vivo. Proc. Natl. Acad. Sci. 93: 8496-8501.

Shen, H., Kan, J.L., Ghigna, C., Biamonti, G., and Green, M.R. 2004a. A single polypyrimidine tract binding protein (PTB) binding site mediates splicing inhibition at mouse IgM exons M1 and M2. RNA 10: 787-794.

Shen, H., Kan, J.L., and Green, M.R. 2004b. Arginine-serine-rich domains bound at splicing enhancers contact the branchpoint to promote prespliceosome assembly. Mol. Cell 13: 367-376. 
Webb et al.

Siebel, C.W. and Guthrie, C. 1996. The essential yeast RNA binding protein Npl3p is methylated. Proc. Natl. Acad. Sci. 93: $13641-13646$.

Sipiczki, M. 2000. Where does fission yeast sit on the tree of life? Genome Biol. 1: reviews1011.1-reviews1011.4.

Smith, C.W. and Valcarcel, J. 2000. Alternative pre-mRNA splicing: The logic of combinatorial control. Trends Biochem. Sci. 25: 381-388.

Spingola, M. and Ares Jr., M. 2000. A yeast intronic splicing enhancer and Nam8p are required for Merlp-activated splicing. Mol. Cell 6: 329-338.

Spingola, M., Grate, L., Haussler, D., and Ares Jr., M. 1999. Genome-wide bioinformatic and molecular analysis of introns in Saccharomyces cerevisiae. RNA 5: 221-234.

Stoilov, P., Daoud, R., Nayler, O., and Stamm, S. 2004. Human tra2- $\beta 1$ autoregulates its protein concentration by influencing alternative splicing of its pre-mRNA. Hum. Mol. Genet. 13: $509-524$.

Sun, Q., Mayeda, A., Hampson, R.K., Krainer, A.R., and Rottman, F.M. 1993. General splicing factor SF2/ASF promotes alternative splicing by binding to an exonic splicing enhancer. Genes \& Dev. 7: 2598-2608.

Takeuchi, N. and Yanagida, M. 1993. A mitotic role for a novel fission yeast protein kinase dsk 1 with cell cycle stage dependent phosphorylation and localization. Mol. Biol. Cell 4: 247-260.

Tanaka, K., Watakabe, A., and Shimura, Y. 1994. Polypurine sequences within a downstream exon function as a splicing enhancer. Mol. Cell. Biol. 14: 1347-1354.

Tang, Z., Kauefer, N.F., and Lin, R.-J. 2002. Interactions between two fission yeast serine/arginine-rich proteins and their modulation by phosphorylation. Biochem. J. 368: 527534.

Tange, T.O. and Kjems, J. 2001. SF2/ASF binds to a splicing enhancer in the third HIV-1 tat exon and stimulates U2AF binding independently of the RS domain. J. Mol. Biol. 312: 649-662.

Tian, M. and Maniatis, T. 1992. Positive control of pre-mRNA splicing in vitro. Science 256: 237-240.

. 1993. A splicing enhancer complex controls alternative splicing of doublesex pre-mRNA. Cell 74: 105-114.

- 1994. A splicing enhancer exhibits both constitutive and regulated activities. Genes \& Dev. 8: 1703-1712.

Tupler, R., Perini, G., and Green, M.R. 2001. Expressing the human genome. Nature 409: 832-833.

Valcarcel, J., Gaur, R.K., Singh, R., and Green, M.R. 1996. Interaction of U2AF65 RS region with pre-mRNA branch point and promotion of base pairing with U2 snRNA [corrected]. Science 273: 1706-1709.

Wang, Z., Hoffmann, H.M., and Grabowski, P.J. 1995. Intrinsic U2AF binding is modulated by exon enhancer signals in parallel with changes in splicing activity. RNA 1: 21-35.

Watakabe, A., Tanaka, K., and Shimura, Y. 1993. The role of exon sequences in splice site selection. Genes \& Dev. 7: 407-418.

Webb, C.J. and Wise, J.A. 2004. The splicing factor U2AF small subunit is functionally conserved between fission yeast and humans. Mol. Cell. Biol. 24: 4229-4240.

Wentz-Hunter, K. and Potashkin, J. 1995. The evolutionary conservation of the splicing apparatus between fission yeast and man. Nucleic Acids Symp. Ser. 33: 226-228.

- 1996. The small subunit of the splicing factor U2AF is conserved in fission yeast. Nucleic Acids Res. 24: 1849-1854.

Wood, V., Gwilliam, R., Rajandream, M.A., Lyne, M., Lyne, R.,
Stewart, A., Sgouros, J., Peat, N., Hayles, J., Baker, S., et al. 2002. The genome sequence of Schizosaccharomyces pombe. Nature 415: 871-880.

Wu, J.Y. and Maniatis, T. 1993. Specific interactions between proteins implicated in splice site selection and regulated alternative splicing. Cell 75: 1061-1070.

$\mathrm{Xu}, \mathrm{R} .$, Teng, J., and Cooper, T.A. 1993. The cardiac troponin $\mathrm{T}$ alternative exon contains a novel purine-rich positive splicing element. Mol. Cell. Biol. 13: 3660-3674.

Zhang, X.H. and Chasin, L.A. 2004. Computational definition of sequence motifs governing constitutive exon splicing. Genes \& Dev. 18: 1241-1250.

Zhu, J. and Krainer, A.R. 2000. Pre-mRNA splicing in the absence of an SR protein RS domain. Genes \& Dev. 14: 3166 3178.

Zuo, P. and Maniatis, T. 1996. The splicing factor U2AF35 mediates critical protein-protein interactions in constitutive and enhancer-dependent splicing. Genes \& Dev. 10: 13561368 . 


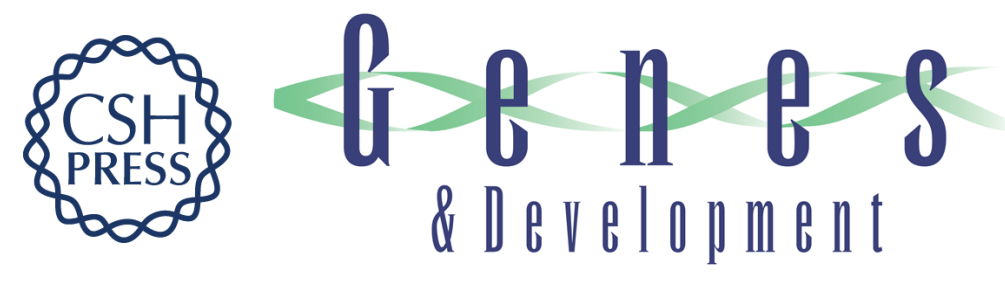

\section{Exonic splicing enhancers in fission yeast: functional conservation demonstrates an early evolutionary origin}

Christopher J. Webb, Charles M. Romfo, Willem J. van Heeckeren, et al.

Genes Dev. 2005, 19:

Access the most recent version at doi:10.1101/gad.1265905

\section{Supplemental http://genesdev.cshlp.org/content/suppl/2004/12/29/gad.1265905.DC1 Material}

References This article cites 98 articles, 56 of which can be accessed free at: http://genesdev.cshlp.org/content/19/2/242.full.html\#ref-list-1

\section{License}

Email Alerting

Receive free email alerts when new articles cite this article - sign up in the box at the top Service

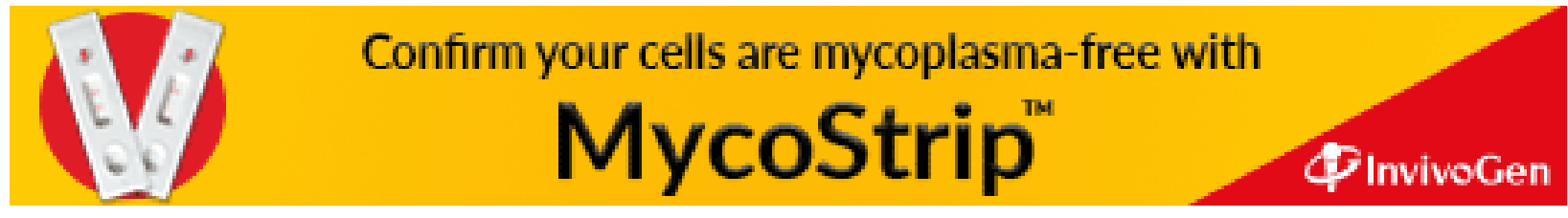

